\title{
OSCILLATORY MHD CONVECTIVE FLOW OF SECOND ORDER FLUID THROUGH POROUS MEDIUM IN A VERTICAL ROTATING CHANNEL IN SLIP-FLOW REGIME WITH HEAT RADIATION
}

\author{
B.P. GARG \\ Devraj Group'sTechnical Campus \\ Ferozpur, Punjab, INDIA \\ K.D. SINGH* \\ Shimla-171003, INDIA \\ E-mail: kdsinghshimla@gmail.com
}

\author{
A.K. BANSAL \\ MIMIT, Malout \\ Punjab, INDIA
}

\begin{abstract}
An analysis of an oscillatory magnetohydrodynamic (MHD) convective flow of a second order (viscoelastic), incompressible, and electrically conducting fluid through a porous medium bounded by two infinite vertical parallel porous plates is presented. The two porous plates with slip-flow condition and the no-slip condition are subjected respectively to a constant injection and suction velocity. The pressure gradient in the channel varies periodically with time. A magnetic field of uniform strength is applied in the direction perpendicular to the planes of the plates. The induced magnetic field is neglected due to the assumption of a small magnetic Reynolds number. The temperature of the plate with no-slip condition is non-uniform and oscillates periodically with time and the temperature difference of the two plates is assumed high enough to induce heat radiation. The entire system rotates in unison about the axis perpendicular to the planes of the plates. Adopting complex variable notations, a closed form solution of the problem is obtained. The analytical results are evaluated numerically and then presented graphically to discuss in detail the effects of different parameters of the problem. The velocity, temperature and the skin-friction in terms of its amplitude and phase angle have been shown graphically to observe the effects of the viscoelastic parameter $\gamma$, rotation parameter $\Omega$, suction parameter $\lambda$, Grashof number Gr, Hartmann number $\mathrm{M}$, the pressure $A$, Prandtl number Pr, radiation parameter $N$ and the frequency of oscillation $\omega$.
\end{abstract}

Key words: slip-flow, convective, porous medium, rotating, oscillatory, radiation, second order fluid.

\section{Introduction}

The wall slip flow is a very important phenomenon that is widely encountered in this era of industrialization. It has numerous applications, for example in lubrication of mechanical devices where a thin film of lubricant is attached to the surface slipping over one another or when the surfaces are coated with special coatings to minimize the friction between them. Marques et al. (2000) considered the effect of the fluid slippage at the plate for Couette flow. Hayat et al. (2008) analyzed slip flow and heat transfer of a second grade fluid past a stretching sheet through a porous space in view of the importance of the slip-flow regime in this era of modern science, technology and its vast ranging industrialization applications. The magnetohydrodynamic (MHD) flow of a viscoelastic fluid as a lubricant is also of primary interest in many industrial applications. The MHD lubrication is an externally pressurized thrust that has been investigated

\footnotetext{
* To whom correspondence should be addressed
} 
both theoretically and experimentally by Maki et al. (1966). Hughes and Elco (1962) investigated the effects of the magnetic field in lubrication. Hamza (1964) considered the squeezing flow between two discs in the presence of a magnetic field. The problem of squeezing flow between rotating discs was studied by Hamza (1991) and Bhattacharya and Pal (1997). Sweet et al. (2011) solved analytically the problem of an unsteady MHD flow of a viscous fluid between moving parallel plates. Hayat et al. (2008) presented analytical solution to the unsteady rotating MHD flow of an incompressible second grade fluid in a porous half space. There is a variety of industrial applications wherein due to high temperature radiative heat is also accompanied along with conduction and convection heat. Algoa et al. (1999) studied the radiative and free convective effects on the MHD flow through a porous medium between infinite parallel plates with timedependent suction. Mebine and Gumus (2010) investigated steady-state solutions to the MHD thermally radiating and reacting thermosolutal flow through a channel with a porous medium.

The flow of viscoelastic fluids through porous media has attracted the attention of a large number of scholars owing to their application in the fields of extraction of energy from geothermal regions and in the flow of oil through porous rocks. Many common liquids such as certain paints, polymer solutions, some organic liquids and many new materials of industrial importance exhibit both viscous and elastic properties. The fluids with such characteristics are called viscoelastic fluids. Flows through the porous medium are also very useful in chemical engineering for the processes of purification and filtration. The scientific treatment of the problem of irrigation, soil erosion etc. are present developments of porous media. Singh (2012) analyzed a viscoelastic mixed convection MHD oscillatory flow through a porous medium filled in a vertical channel. Rahman and Sarkar (2004) investigated the unsteady MHD flow of a viscoelastic Oldroyd fluid under time varying body forces through a rectangular channel. Singh and Singh (1966) studied the MHD flow of a dusty viscoelastic (Oldroyd B-liquid) through a porous medium between two parallel plates inclined to horizon. Rajgopal and Gupta (1984) obtained an exact solution for the flow of a non-Newtonian fluid past an infinite porous plate. Applying quasilinearization to the problem Verma et al. (1984) analyzed the steady laminar flow of a second grade fluid between two rotating porous disks. Rhodes and Rouleau (1966) studied the hydrodynamic lubrication of partial porous metal bearings. Mehmood and Ali (2007) extended the problem of the oscillatory MHD flow in a channel filled with porous medium studied by Makinde and Mhone (2005) to slip-flow regime. Further by applying the perturbation technique Kumar et al. (2010) investigated the same problem of slip-flow regime for the unsteady MHD periodic flow of a viscous fluid through a planer channel. Very recently, Choudhury and Das (2012) studied the combined effects of the magnetic field and heat radiation on a viscoelastic flow in a channel filled with a porous medium. This study is also an extension of the paper by Makinde and Mhone (2005). Singh and Devi (2010) studied the effect of slip velocity on the MHD oscillatory flow through a porous medium in a channel. Sivaraj and Kumar (2011) analyzed the unsteady MHD oscillatory chemically reacting slip-flow in a planer channel with varying concentration. Hamza et al. (2011) investigated the effects of slip condition on an unsteady heat transfer to the MHD oscillatory flow through a porous medium. Singh and Kumar (2011) studied analytically the fluctuating heat and mass transfer MHD free convection flow of a radiating and reacting fluid past a vertical porous plate in slip-flow regime.

The objective of the present analysis is to study an oscillatory convection flow of an incompressible, electrically conducting second order fluid in a vertical porous channel. Constant injection and suction is applied at the left and the right infinite porous plates respectively. The entire system rotates about an axis perpendicular to the planes of the plates and a uniform magnetic field is also applied along this axis of rotation. A general exact solution of the partial differential equations governing the flow problem is obtained and the effects of various flow parameters on the velocity field and the skin friction are discussed in the last section of the paper with the help of figures.

\section{Basic equations}

Consider a flow of a second order (viscoelastic), incompressible and electrically conducting fluid in a rotating vertical channel. In order to derive the basic equations for the problem under consideration the following assumptions are made: 
(i) The two infinite vertical parallel plates of the channel are permeable and electrically non-conducting.

(ii) The vertical channel is filled with a porous medium.

(iii) The flow considered is fully developed, laminar and oscillatory.

(iv) The fluid is second order, incompressible and finitely conducting.

(v) All fluid properties are assumed to be constant except that of the influence of density variation with temperature is considered only in the body force term.

(vi) The pressure gradient in the channel oscillates periodically with time.

(vii) A magnetic field of uniform strength $B_{0}$ is applied perpendicular to the plates of the channel.

(viii) The magnetic Reynolds number is taken to be small enough so that the induced magnetic field is neglected.

(ix) Hall effect, electrical and polarization effects are also neglected.

(x) The temperature of the plate with no-slip condition is non-uniform and oscillates periodically with time.

(xi) The temperature difference of the two plates is also assumed to be high enough to induce heat transfer due to radiation.

(xii) The fluid is assumed to be optically thin with relatively low density.

(xiii) The entire system (consisting of channel plates and the fluid) rotates about an axis perpendicular to the plates.

Under these assumptions we write hydromagnetic governing equations of motion and continuity in a rotating frame of reference as

$$
\begin{aligned}
& \nabla \cdot \boldsymbol{V}=0, \\
& \rho\left(\frac{\partial \boldsymbol{V}}{\partial t}+(\boldsymbol{V} \cdot \nabla \boldsymbol{V})+2 \mathbf{\Omega} \times \boldsymbol{V}\right)=\frac{\boldsymbol{\mu}}{\boldsymbol{K}^{*}} \boldsymbol{V}+\nabla \cdot \exists+(\boldsymbol{J} \times \boldsymbol{B})+\boldsymbol{F}, \\
& \rho c_{p}\left[\frac{\partial T}{\partial t}+(\boldsymbol{V} \cdot \nabla) T\right]=k \nabla^{2} T-\nabla q .
\end{aligned}
$$

In Eq.(2.2) the last term on the left hand side is the Coriolis force. On the right hand side of Eq.(2.2) the last term $\boldsymbol{F}\left(=\rho g \beta T^{*}\right)$ accounts for the force due to buoyancy. The last but one term is the Lorentz Force due to the magnetic field $\boldsymbol{B}$ and is given by

$$
\boldsymbol{J} \times \boldsymbol{B}=\sigma(\boldsymbol{V} \times \boldsymbol{B}) \times \boldsymbol{B},
$$

and the modified pressure $p^{*}=p^{\prime}-\frac{\rho}{2}|\boldsymbol{\Omega} \times \boldsymbol{R}|^{2}$ where $\boldsymbol{R}$ denotes the position vector from the axis of rotation, $p^{\prime}$ denotes the fluid pressure, $\boldsymbol{J}$ is the current density, and all other quantities have their usual meaning and have been defined from time to time in the text.

In the second term of the right hand side of Eq.(2.2), $\exists$ is the Cauchy stress tensor and the constitutive equation derived by Coleman and Noll (1960) for an incompressible homogeneous fluid of second order is

$$
\exists=-p_{1} \boldsymbol{I}+\mu_{1} \boldsymbol{A}_{1}+\mu_{2} \boldsymbol{A}_{2}+\mu_{3} \boldsymbol{A}_{1}^{2}
$$

Here $-p_{1} I$ is the interdeterminate part of the stress due to constraint of incompressibility, $\mu_{1}, \mu_{2}$ and $\mu_{3}$ are the material constants describing viscosity, elasticity and cross-viscosity respectively. The kinematic $A_{l}$ and $A_{2}$ are the Rivelen Ericson constants are defined as 


$$
\boldsymbol{A}_{l}=(\nabla \boldsymbol{V})+(\nabla \boldsymbol{V})^{T}, \quad \boldsymbol{A}_{2}=\frac{d \boldsymbol{A}_{1}}{d t}+(\nabla \boldsymbol{V})^{T} \boldsymbol{A}_{l}+\boldsymbol{A}_{l}(\nabla \boldsymbol{V})
$$

where $\nabla$ denotes the gradient operator and $d / d t$ the material time derivative. According to Markovitz and Coleman (1964) the material constants $\mu_{1}, \mu_{3}$ are taken as positive and $\mu_{2}$ as negative.

\section{Formulation of the problem}

In the present analysis, we consider an unsteady flow of a second order (viscoelastic), incompressible and electrically conducting fluid bounded by two infinite vertical porous plates distance ' $d$ ' apart. A coordinate system is chosen such that the $X^{*}$-axis is oriented upward along the centerline of the channel and $Z^{*}$-axis taken perpendicular to the planes of the plates lying in $z^{*}= \pm \frac{d}{2}$ planes. The fluid is injected through the porous plate at $z^{*}=-\frac{d}{2}$ with constant velocity $w_{0}$ and simultaneous sucked through the other porous plate at $z^{*}= \pm \frac{d}{2}$ with the same velocity $w_{0}$. The non-uniform temperature of the plate at $z^{*}= \pm \frac{d}{2}$ is assumed to be varying periodically with time.

The temperature difference between the plates is high enough to induce the heat due to radiation. The $Z^{*}$ - axis is considered to be the axis of rotation about which the fluid and the plates are assumed to be rotating as a solid body with a constant angular velocity $\Omega^{*}$. A transverse magnetic field of uniform strength $B\left(0,0, B_{0}\right)$ is also applied along the axis of rotation. All physical quantities depend on $z^{*}$ and $t^{*}$ only for this problem of fully developed laminar flow. A schematic diagram of the flow problem is shown in Fig.1.

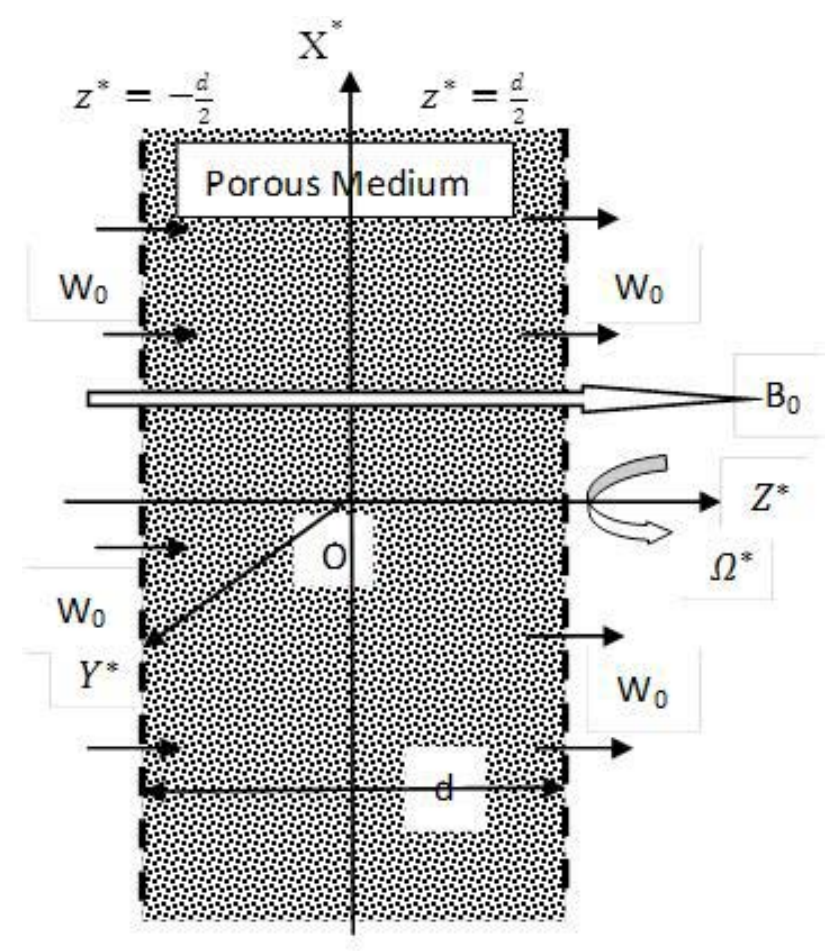

Fig.1. Physical configuration of the flow. 
On integration of the equation of continuity $\nabla \cdot \boldsymbol{V}=0$ we get $w^{*}=w_{0}$. Then the velocity may reasonably be assumed with its components along $x^{*}, y^{*}, z^{*}$ directions as $\boldsymbol{V}\left(u^{*}, v^{*}, w_{0}\right)$. Under the usual Boussinesq approximation and following Attia and Ewis (2010) the MHD flow in the rotating channel is governed by the following Cartesian equations

$$
\begin{aligned}
& \frac{\partial u^{*}}{\partial t^{*}}+w_{0} \frac{\partial u^{*}}{\partial z^{*}}=-\frac{1}{\rho} \frac{\partial p^{*}}{\partial x^{*}}+\vartheta_{1} \frac{\partial^{2} u^{*}}{\partial z^{* 2}}+\vartheta_{2} \frac{\partial^{3} u^{*}}{\partial z^{* 2} \partial t^{*}}+2 \Omega^{*} v^{*}-\frac{\sigma B_{0}^{2}}{\rho} u^{*}-\frac{\vartheta_{1} u^{*}}{K^{*}}+g \beta\left(T^{*}-T_{1}\right) \\
& \frac{\partial v^{*}}{\partial t^{*}}+w_{0} \frac{\partial v^{*}}{\partial z^{*}}=-\frac{1}{\rho} \frac{\partial p^{*}}{\partial y^{*}}+\vartheta_{1} \frac{\partial^{2} v^{*}}{\partial z^{* 2}}+\vartheta_{2} \frac{\partial^{3} v^{*}}{\partial z^{* 2} \partial t^{*}}-2 \Omega^{*} u^{*}-\frac{\sigma B_{0}^{2}}{\rho} v^{*}-\frac{\vartheta_{1} u^{*}}{K^{*}} \\
& 0=-\frac{1}{\rho} \frac{\partial p^{*}}{\partial z^{*}} \\
& \frac{\partial T^{*}}{\partial t^{*}}+w_{0} \frac{\partial T^{*}}{\partial z^{*}}=\frac{k}{\rho c_{p}} \frac{\partial^{2} T^{*}}{\partial z^{* 2}}-\frac{1}{\rho c_{p}} \frac{\partial q}{\partial z^{*}}
\end{aligned}
$$

where $\rho$ is the density, $\vartheta_{l}$ is the kinematic viscosity, $\vartheta_{2}$ is viscoelasticity, $p^{*}$ is the modified pressure, $t^{*}$ is the time, $\sigma$ is the electric conductivity, $B_{0}$ is the component of the applied magnetic field along the $z^{*}$-axis, $g$ is the acceleration due to gravity, $k$ is the thermal conductivity, $c_{p}$ is the specific heat at constant pressure and the last term in Eq.(3.4) is the radiative heat flux.

Following Cogley et al. (1968) it is assumed that the fluid is optically thin with a relatively low density and the heat flux due to radiation in Eq.(3.4) is given by

$$
\frac{\partial q}{\partial z^{*}}=4 \alpha^{2}\left(T^{*}-T_{1}\right)
$$

where $\alpha$ is the mean radiation absorption coefficient. After the substitution of Eq.(3.5), Eq.(3.4) becomes

$$
\frac{\partial T^{*}}{\partial t^{*}}+w_{0} \frac{\partial T^{*}}{\partial z^{*}}=\frac{k}{\rho c_{p}} \frac{\partial^{2} T^{*}}{\partial z^{* 2}}-\frac{4 \alpha^{2}}{\rho c_{p}}\left(T^{*}-T_{1}\right) .
$$

Equation (3.3) shows the constancy of the hydrodynamic pressure along the axis of rotation. We shall assume now that the fluid flows under the influence of pressure gradient varying periodically with time in the $X^{*}$-axis is of the form

$$
-\frac{1}{\rho} \frac{\partial p^{*}}{\partial x^{*}}=A \cos \omega^{*} t^{*} \text { and } \quad-\frac{1}{\rho} \frac{\partial p^{*}}{\partial y^{*}}=0, \text { where } A \text { is a constant. }
$$

The boundary conditions for the problem are

$$
z^{*}=\frac{d}{2}: \quad u^{*}=v^{*}=0, \quad T^{*}=T_{1}+\left(T_{2}-T_{1}\right) \cos \omega^{*} t^{*}
$$




$$
z^{*}=-\frac{d}{2} ; \quad u^{*}=L_{1} \frac{\partial u^{*}}{\partial z^{*}}, \quad v^{*}=L_{1} \frac{\partial v^{*}}{\partial z^{*}}, \quad T^{*}=T_{1}
$$

where $\omega^{*}$ is the frequency of oscillations, $L_{l}=\left(\frac{2-r_{l}}{r_{l}}\right) L, L$ being mean free path and $r_{l}$ is the Maxwell's reflexion coefficient.

Introducing the following non-dimensional quantities

$$
\begin{aligned}
& \eta=\frac{z^{*}}{d}, \quad x=\frac{x^{*}}{d}, \quad y=\frac{y^{*}}{d}, \quad u=\frac{u^{*}}{w_{0}}, \quad v=\frac{v^{*}}{w_{0}}, \\
& T=\frac{T^{*}-T_{1}}{T_{2}-T_{1}}, \quad t=\frac{t^{*} w_{0}}{d}, \quad \omega=\frac{\omega^{*} d}{w_{0}}, \quad p=\frac{p^{*}}{\rho w_{0}^{2}}
\end{aligned}
$$

into Eqs (3.1), (3.2) and (3.6), we get

$$
\begin{aligned}
& \lambda\left(\frac{\partial u}{\partial t}+\frac{\partial u}{\partial \eta}\right)=-\lambda \frac{\partial p}{\partial x}+\frac{\partial^{2} u}{\partial \eta^{2}}+\gamma \frac{\partial^{3} u}{\partial \eta^{2} \partial t}+2 \Omega v-M^{2} u-K^{-1} u+\text { Gr } T \\
& \lambda\left(\frac{\partial v}{\partial t}+\frac{\partial v}{\partial \eta}\right)=-\lambda \frac{\partial p}{\partial y}+\frac{\partial^{2} v}{\partial \eta^{2}}+\gamma \frac{\partial^{3} v}{\partial \eta^{2} \partial t}-2 \Omega u-M^{2} v-K^{-1} v \\
& \lambda \operatorname{Pr}\left(\frac{\partial T}{\partial t}+\frac{\partial T}{\partial \eta}\right)=\frac{\partial^{2} T}{\partial \eta^{2}}-N^{2} T
\end{aligned}
$$

where '*' represents the dimensional physical quantities,

$\lambda=\frac{w_{0} d}{\vartheta_{1}}$ is the injection/suction parameter,

$\gamma=\frac{v_{2} \lambda}{d^{2}}$ is the visco-elastic parameter,

$\Omega=\frac{\Omega^{*} d^{2}}{\vartheta_{1}}$ is the rotation parameter,

$\mathrm{M}=B_{0} d \sqrt{\frac{\sigma}{\rho \vartheta_{l}}}$ is the Hartmann number,

$K=\frac{K^{*}}{d^{2}}$ is the permeability of the porous medium,

$\mathrm{Gr}=\frac{g \beta d^{2}\left(T_{2}-T_{1}\right)}{\vartheta_{1} w_{0}}$ is the Grashof number,

$\operatorname{Pr}=\frac{\rho \vartheta_{1} c_{p}}{k}$ is the Prandtl number, 
$N=\frac{2 \alpha d}{\sqrt{k}}$ is the radiation parameter,

$\omega=\frac{\omega^{*} d}{w_{0}}$ is the frequency of oscillations.

The boundary conditions in the dimensionless form become

$$
\begin{aligned}
& \eta=\frac{1}{2}: \quad u=v=0, \quad T=\cos \omega t, \\
& \eta=-\frac{1}{2}: \quad u=\delta \frac{\partial u}{\partial \eta}, \quad v=\delta \frac{\partial v}{\partial \eta}, \quad T=0
\end{aligned}
$$

where $\delta=L_{1} / d$ is the dimensionless slip-flow parameter.

For the oscillatory internal flow we shall assume that the fluid flows under the influence of a nondimension pressure gradient varying periodically with time in the direction of the $X$-axis only which implies that

$$
-\frac{\partial p}{\partial x}=A \cos \omega t, \quad \text { and } \quad-\frac{\partial p}{\partial y}=0
$$

\section{Solution of the problem}

Now combining Eqs (3.11) and (3.12) into a single equation by introducing a complex function of the form $F=u+i v$ and with the help of Eqs (3.16), we get

$$
\lambda\left(\frac{\partial F}{\partial t}+\frac{\partial F}{\partial \eta}\right)=-\lambda \frac{\partial p}{\partial x}+\frac{\partial^{2} F}{\partial \eta^{2}}+\gamma \frac{\partial^{3} F}{\partial \eta^{2} \partial t}-\left(M^{2}+K^{-1}+2 i \Omega\right) F+\operatorname{Gr} T,
$$

with corresponding boundary conditions as

$$
\begin{aligned}
& \eta=\frac{1}{2}: \quad F=0, \quad T=\cos \omega t, \\
& \eta=-\frac{1}{2}: \quad F=\delta \frac{\partial F}{\partial \eta}, \quad T=0 .
\end{aligned}
$$

In order to solve Eqs (4.1) and (4.2) under boundary conditions (4.2) and (4.3) it is convenient to adopt complex notations for the velocity, temperature and the pressure as

$$
F(\eta, t)=F_{0}(\eta) e^{i \omega t}, \quad T=\theta_{0}(\eta) e^{i \omega t}, \quad-\frac{\partial p}{\partial x}=A e^{i \omega t}
$$

The solutions will be obtained in terms of complex notations, the real part of which will have physical significance.

The boundary conditions (4.2) and (4.3) in complex notations can also be written as 


$$
\begin{aligned}
& \eta=\frac{1}{2}: \quad F=0, \quad T=e^{i \omega t}, \\
& \eta=-\frac{1}{2}: \quad F=\delta \frac{\partial F}{\partial \eta}, \quad T=0 .
\end{aligned}
$$

Substituting expressions (4.4) in Eqs (4.1) and (3.13), we get

$$
\begin{aligned}
& (1+i \omega \gamma) \frac{d^{2} F_{0}}{d \eta^{2}}-\lambda \frac{d F_{0}}{d \eta}-\left(M^{2}+K^{-1}+2 i \Omega+i \omega \lambda\right) F_{0}=-\lambda A-\operatorname{Gr} \theta_{0} \\
& \frac{d^{2} \theta_{0}}{d \eta^{2}}-\lambda \operatorname{Pr} \frac{d \theta_{0}}{d \eta}-\left(N^{2}+i \omega \lambda \operatorname{Pr}\right) \theta_{0}=0 .
\end{aligned}
$$

The transformed boundary conditions reduce to

$$
\begin{aligned}
& \eta=\frac{1}{2}: \quad F_{0}=0, \quad \theta_{0}=1, \\
& \eta=-\frac{1}{2}: \quad F_{0}=\delta \frac{\partial F_{0}}{\partial \eta}, \quad \theta_{0}=0 .
\end{aligned}
$$

The solution of the ordinary differential Eq.(4.7) under the boundary conditions (4.9) and (4.10) gives the velocity field as

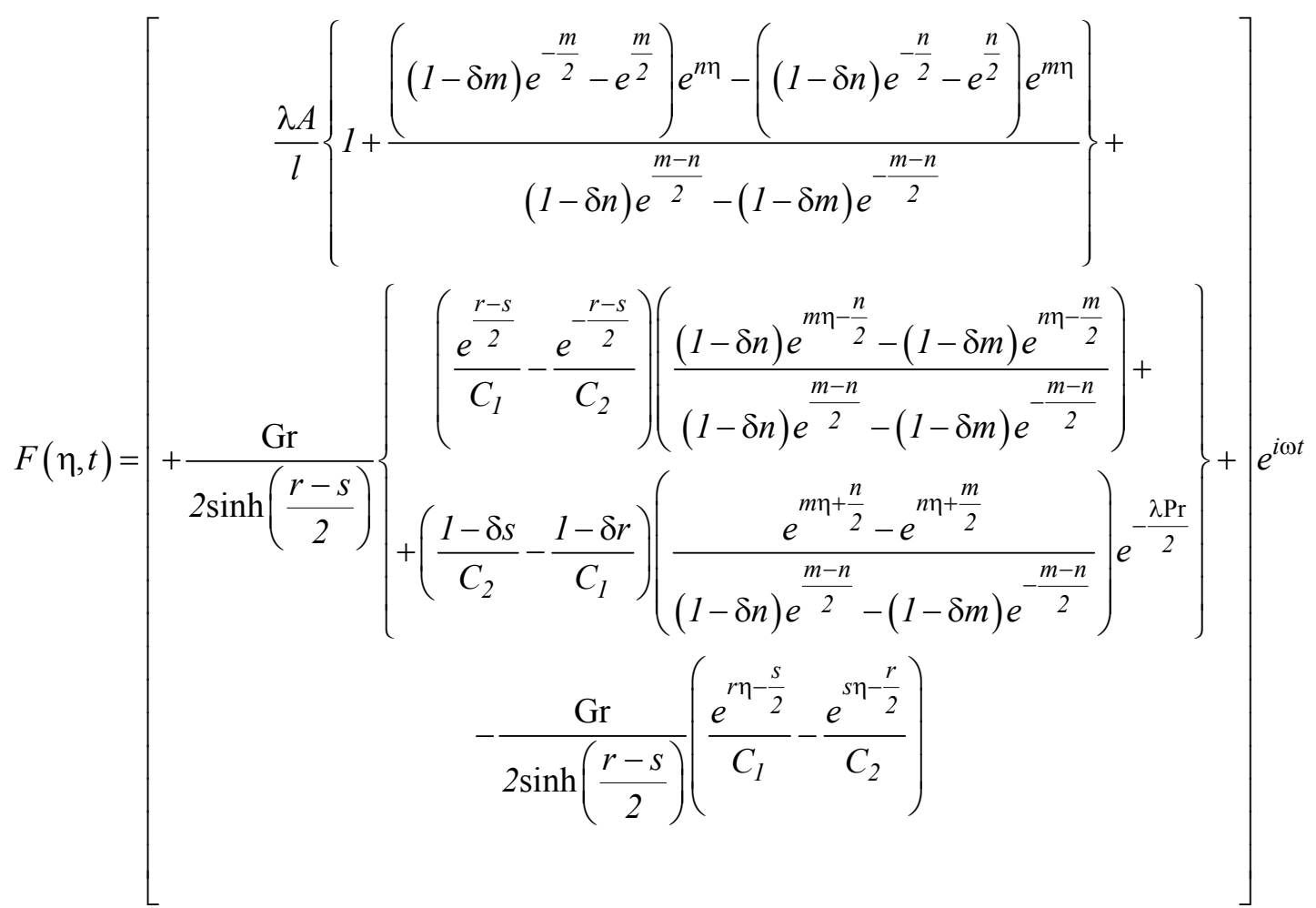


where $l=\left(M^{2}+K^{-1}+2 i \Omega+i \omega \lambda\right), C_{1}=(1+i \omega \gamma) r^{2}-\lambda r-l, C_{2}=(1+i \omega \gamma) s^{2}-\lambda s-l$,

$$
\begin{array}{ll}
m=\frac{\lambda+\sqrt{\lambda^{2}+4 l(1+i \omega \gamma)}}{2(1+i \omega \gamma)}, \quad n=\frac{\lambda-\sqrt{\lambda^{2}+4 l(1+i \omega \gamma)}}{2(1+i \omega \gamma)}, \\
r=\frac{\lambda \operatorname{Pr}+\sqrt{\lambda^{2} \operatorname{Pr}^{2}+4\left(N^{2}+i \omega \lambda \operatorname{Pr}\right)}}{2}, \quad s=\frac{\lambda \operatorname{Pr}-\sqrt{\lambda^{2} \operatorname{Pr}^{2}+4\left(N^{2}+i \omega \lambda \operatorname{Pr}\right)}}{2} .
\end{array}
$$

Similarly, the solution of Eq.(4.8) for the temperature field can be obtained under the boundary conditions (4.9) and (4.10) as

$$
T(\eta, t)=\left(\frac{e^{r \eta-\frac{s}{2}}-e^{s \eta-\frac{r}{2}}}{2 \sinh \left(\frac{r-s}{2}\right)}\right) e^{i \omega t} .
$$

From the velocity field obtained in Eq.(4.11) we can get the skin-friction $\tau_{L}$ at the left plate $(\eta=-$ $0.5)$ in terms of its amplitude $|F|$ and phase angle $\varphi$ as

$$
\tau=|F| \cos (t+\varphi), \text { with }
$$

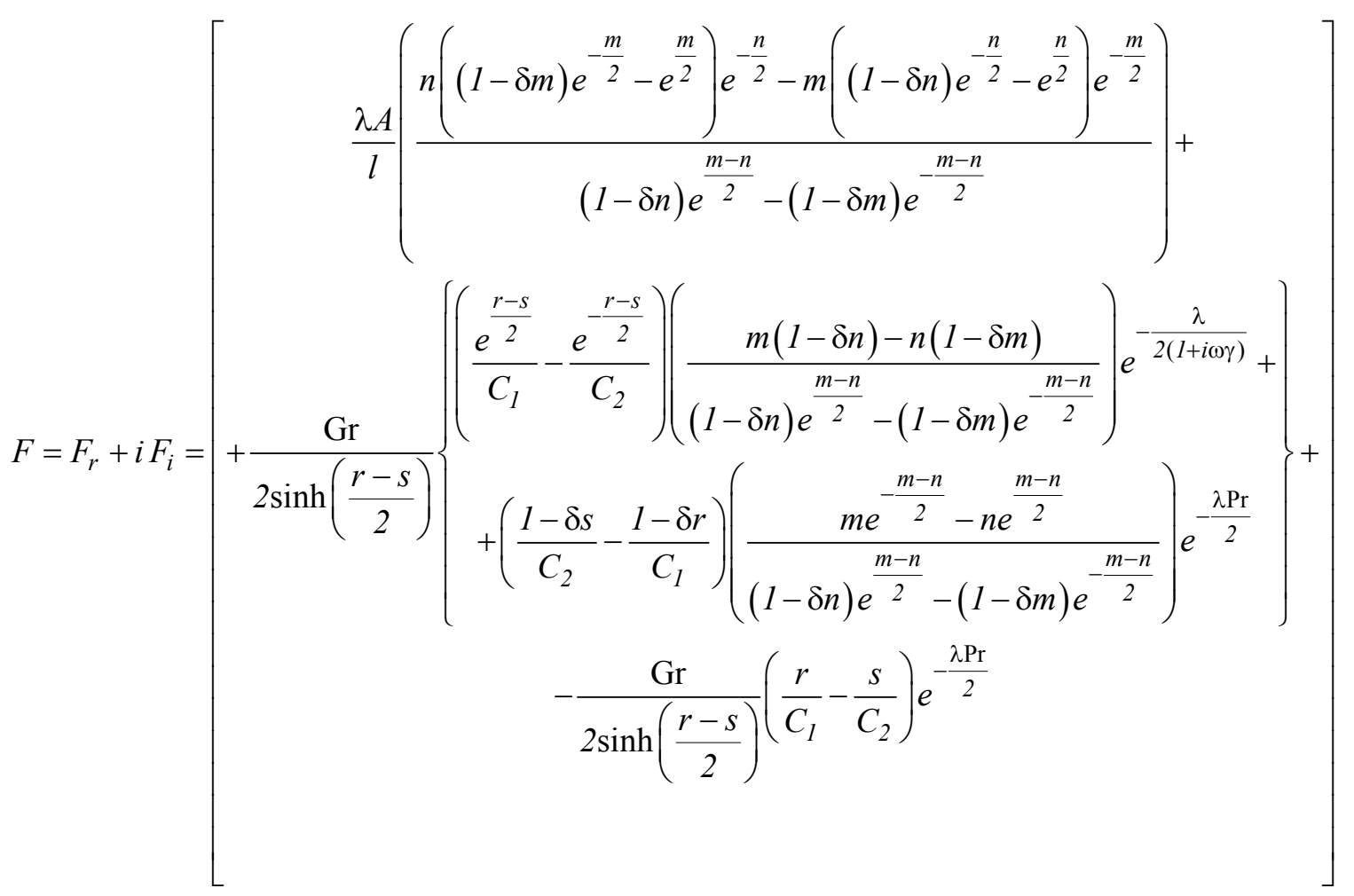


The amplitude is $|F|=\sqrt{F_{r}^{2}+F_{i}^{2}}$ and the phase angle is $\varphi=\tan ^{-1} \frac{F_{i}}{F_{r}}$.

Similarly the Nusselt number $\mathrm{Nu}$ in terms of its amplitude $|H|$ and the phase angle $\psi$ can be obtained from Eq.(4.12) for the temperature field as

$$
\begin{aligned}
& q=|H| \cos (t+\psi), \\
& \text { with }=H r+i H i=\frac{(r-s) e^{-\frac{\lambda \operatorname{Pr}}{2}}}{2 \sinh \left(\frac{r-s}{2}\right)}
\end{aligned}
$$

where the amplitude $|H|$ and the phase angle $\psi$ of the rate of heat transfer are given as

$$
|H|=\sqrt{H r^{2}+H i^{2}}, \quad \psi=\tan ^{-1} \frac{H i}{H r} .
$$

The temperature field, amplitude and phase of the Nusselt number need no further discussion because these have already been discussed in detail by Singh (2011).

\section{Results and discussions}

The problem of an oscillatory magnetohydrodynamic (MHD) convective and radiative flow in a vertical porous channel is analyzed in slip-flow regime. The viscoelastic, incompressible and electrically conducting fluid is injected through one of the porous plates and simultaneously removed through the other porous plate with the same velocity. The entire system (consisting of porous channel plates and the fluid) rotates about an axis perpendicular to the plates. The closed form solutions for the velocity and temperature fields are obtained analytically and then evaluated numerically for different values of parameters which appeared in the equations. To have a better insight into the physical problem the variations of the velocity, the skin-friction in terms of its amplitude and phase angle are evaluated numerically for different sets of the values of the injection/suction parameter $\lambda$, viscoelastic parameter $\gamma$, rotation parameter $\Omega$, Hartmann number $\mathrm{M}$, permeability parameter $K$, Grashof number Gr, Prandtl number Pr, radiation parameter $N$, pressure gradient $A$ and the frequency of oscillations $\omega$. These numerical values are then shown graphically to assess the effect of each parameter for the two cases of rotation $\Omega=5$ (small) and $\Omega=15$ (large). The velocity variations are presented graphically in Figs 2 to 11 for $\gamma=0.2, \lambda=0.5, \mathrm{Gr}=5, \mathrm{M}=2, K=0.2, \operatorname{Pr}=0.7$, $N=1, A=5, \omega=5$ and $t=0$ except the ones shown in these figures.

Figure 2 illustrates the variation of the velocity with the increasing slip-flow parameter $\delta$. It is quite obvious from this figure that velocity goes on increasing with increasing slip-flow parameter $\delta$. The velocity variations with the rotation parameter $\Omega$ are presented in Fig.3.The velocity decreases with increasing rotation $\Omega$ of the entire system. The velocity profiles initially remain parabolic for small values of the rotation parameter $\Omega$ with maximum at the centre of the channel and then as rotation increases the velocity profiles flatten. The variations of the velocity profiles with the viscoelastic parameter $\gamma$ are presented in Fig.4. It is very clear from this figure that the velocity decreases with the increase of the viscoelastic parameter for both the cases of small $(\Omega=5)$ and large $(\Omega=15)$ rotations of the channel. However, for both these cases of rotation, the velocity increases significantly with increasing the injection/suction parameter $\lambda$ as is revealed in Fig.5. The variations of the velocity profiles with the Grashof number Gr is shown in Fig.6. 
Although the velocity for large rotation $(\Omega=15)$ is much less than the velocity for small rotation $(\Omega=5)$, in both these cases velocity increases with the increasing Grashof number Gr.

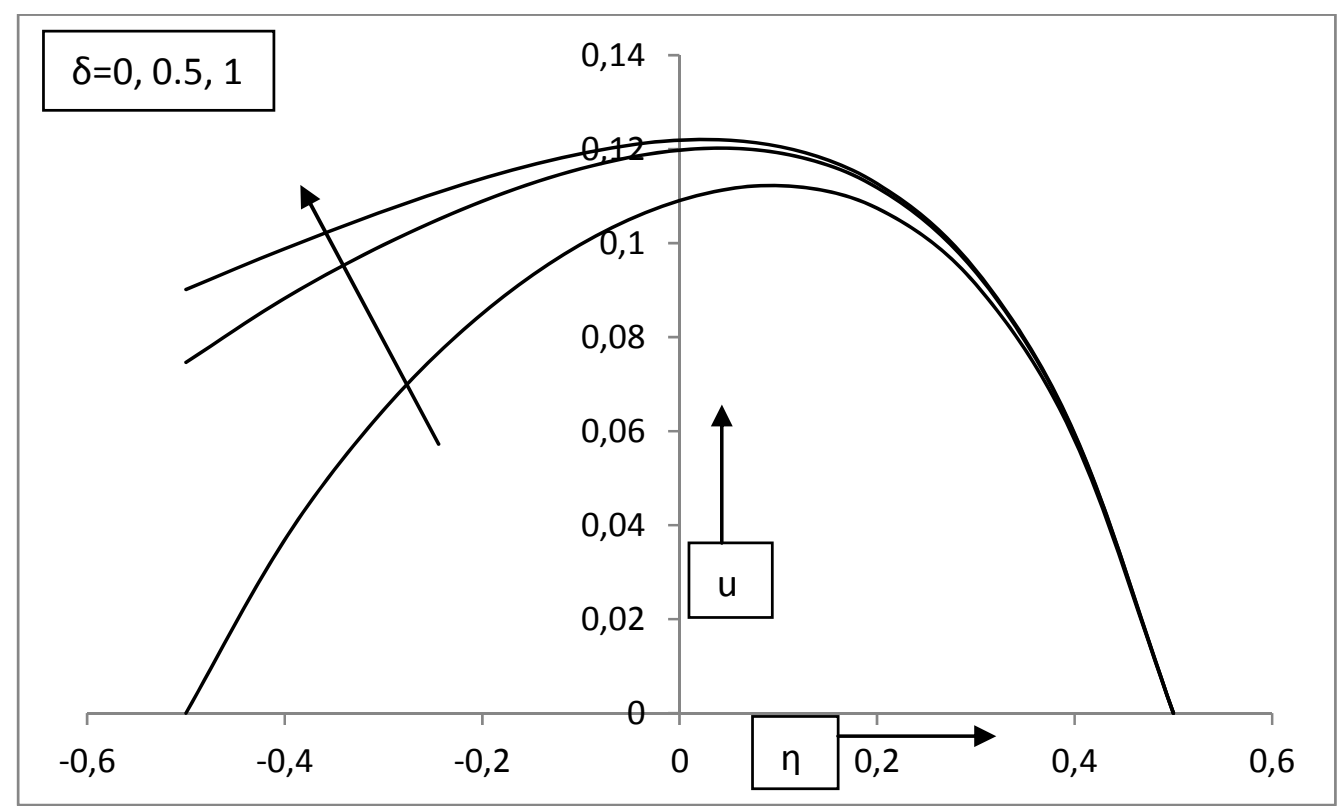

Fig.2. Velocity variations with slip-flow parameter $\delta$.

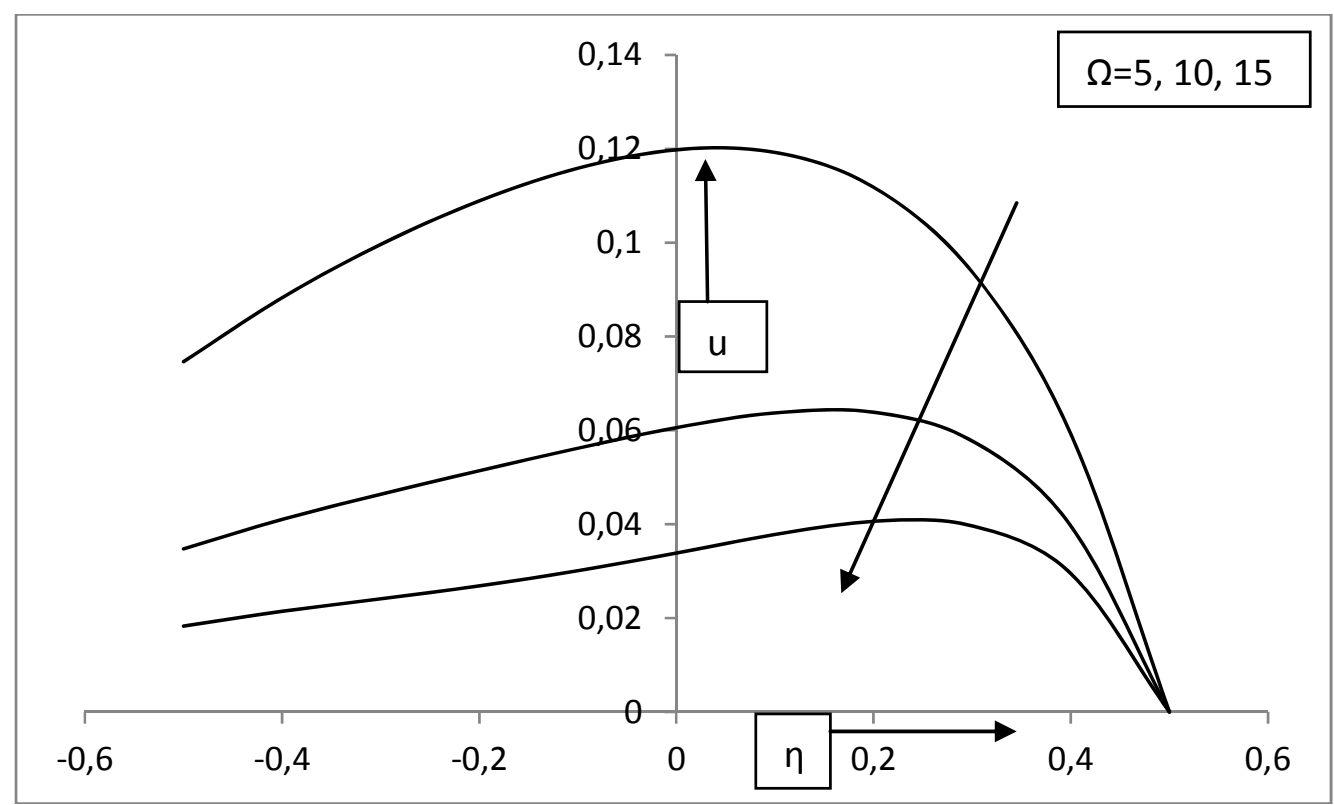

Fig.3.Velocity variations with rotation parameter $\Omega$. 


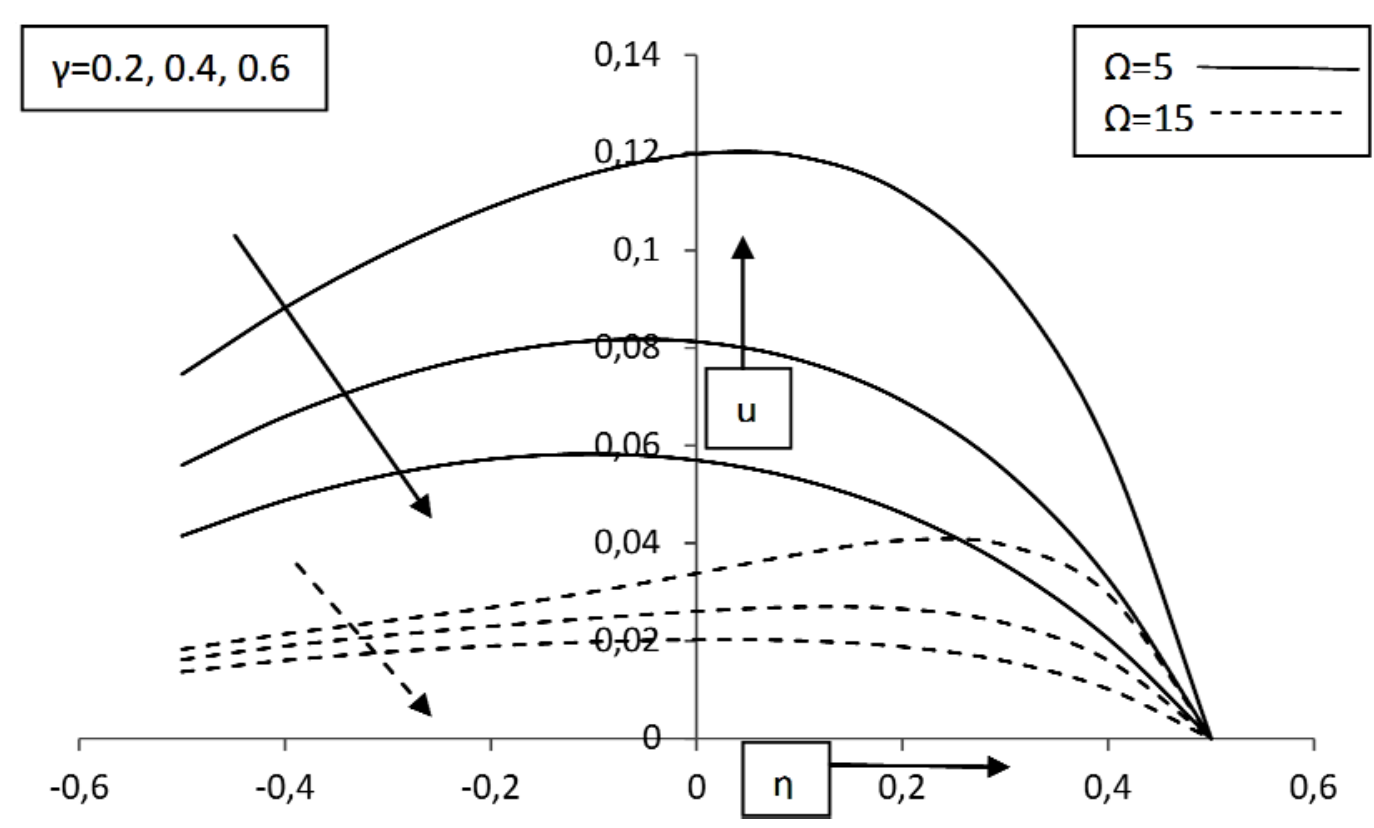

Fig.4. Velocity variations with viscoelastic parameter $\gamma$.

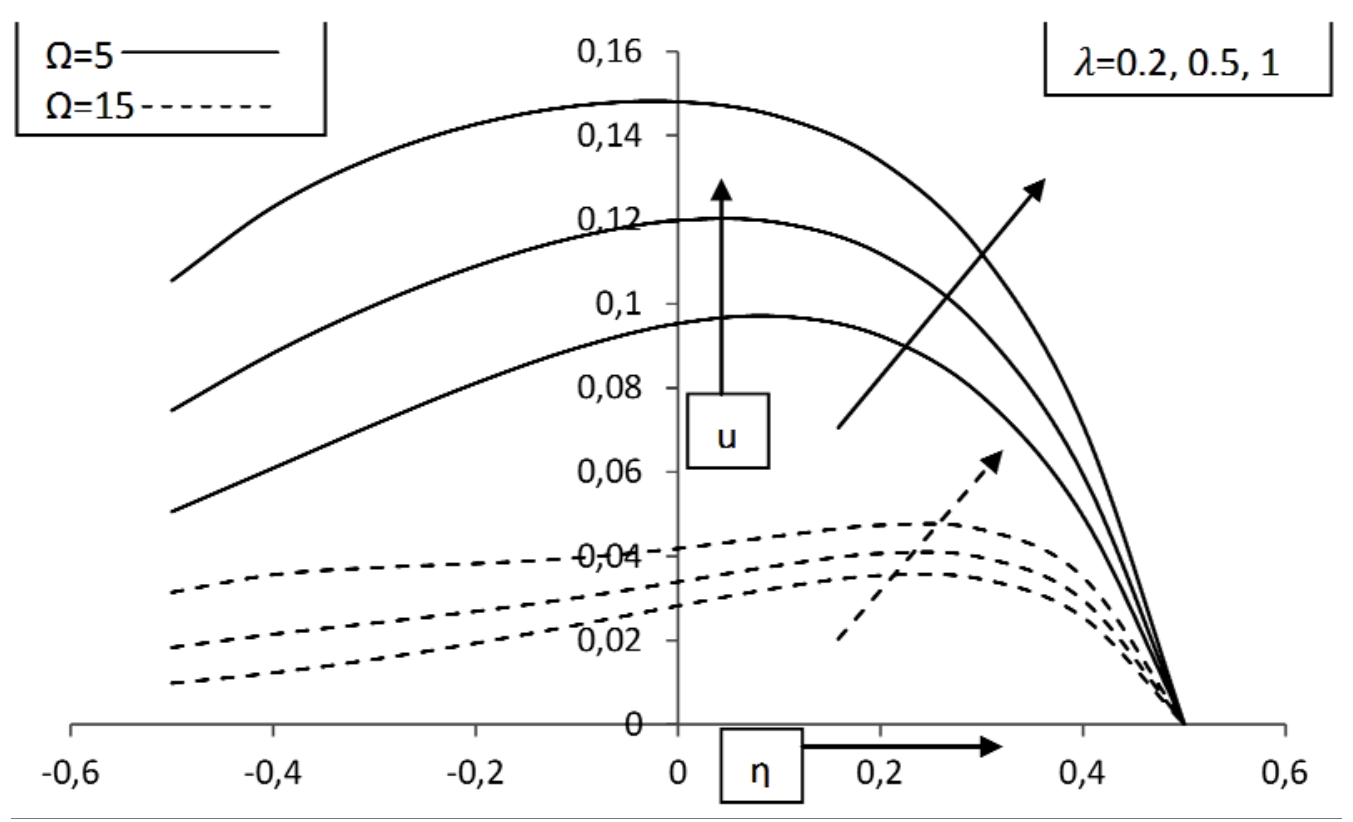

Fig.5. Velocity variations with injection/suction parameter $\lambda$. 


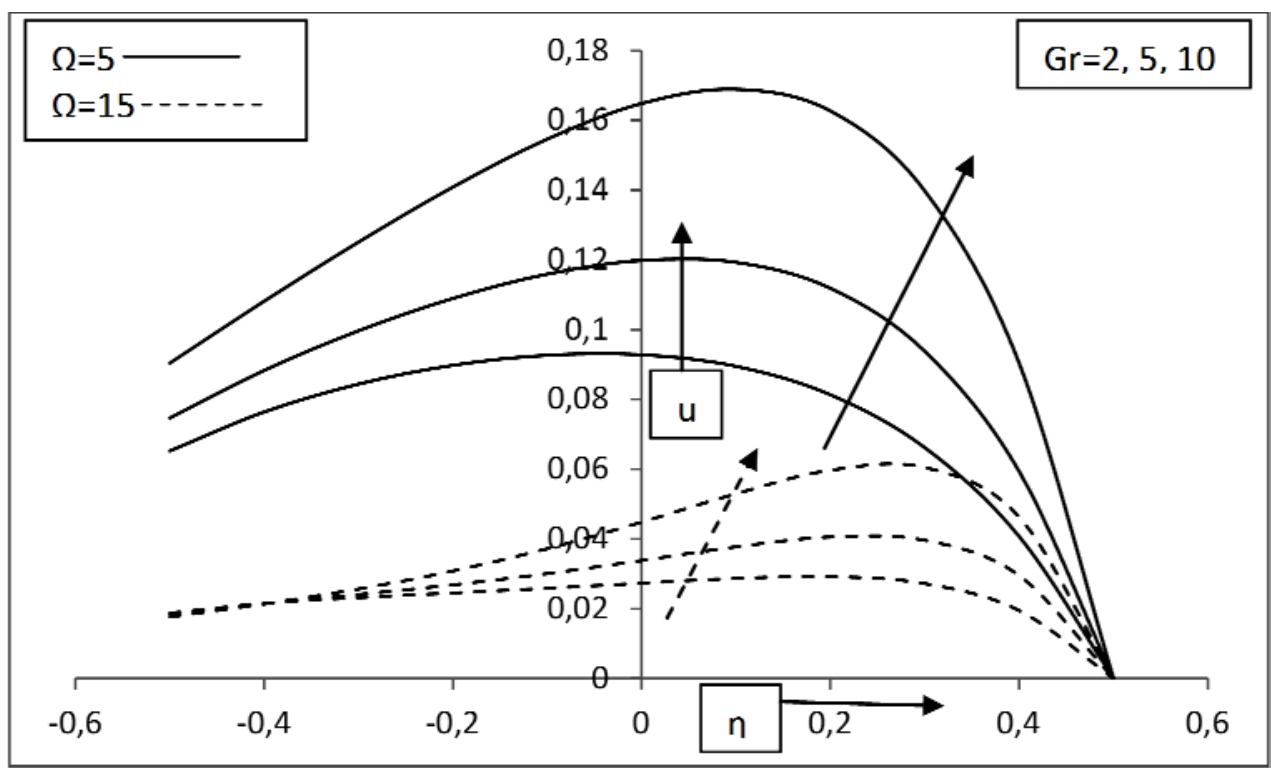

Fig.6. Velocity variations with Grashof number Gr.

The variation of the velocity profiles with the Hartmann number $M$ is presented in Fig.7. For both cases of small $\Omega(=5)$ and large $\Omega(=15)$ rotations the velocity goes on increasing with increasing $M$. This means that the backward flow caused by the rotation of the system and the viscoelasticity of the fluid is resisted by the increasing Lorentz force due to the increasing magnetic field strength. Figure 8 depicts the variations of the velocity with the permeability of the porous medium $K$. It is observed from the figure that the velocity decreases with the increasing permeability $K$ of the porous medium for both small $(\Omega=5)$ and large $(\Omega=15)$ rotations of this system of the viscoelastic fluid flow. We also find from Fig.9 and Fig.10 respectively that with the increase of the Prandtl number Pr and the radiation parameter $N$ the velocity decreases both for small $(\Omega=5)$ and large $(\Omega=15)$ rotations. From Fig.11 it is evident that the velocity goes on increasing with the increasing favorable pressure gradient $P(>0)$. The effects of the frequency of oscillations $\omega$ on velocity are exhibited in Fig.12. It is noticed that velocity decreases with the increasing frequency $\omega$ for either case of channel rotation, large or small.

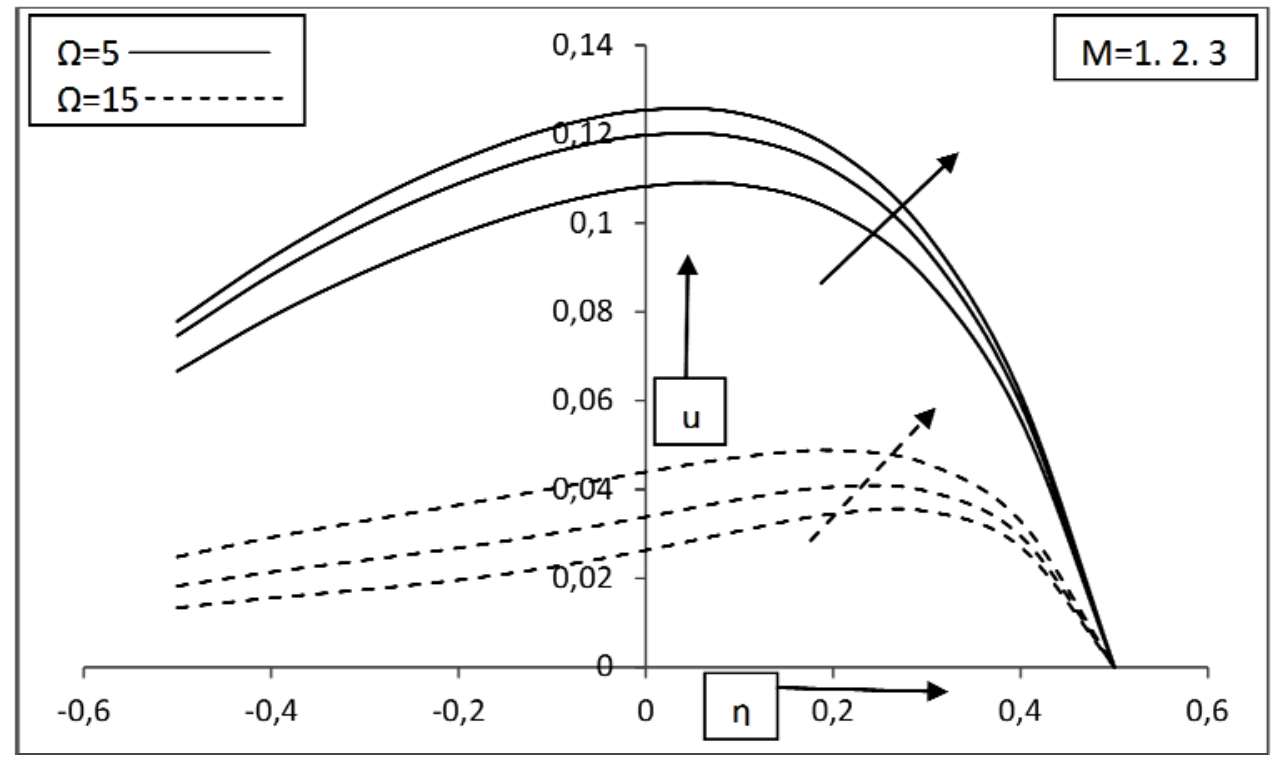

Fig.7. Velocity variations with Hartmann number M. 


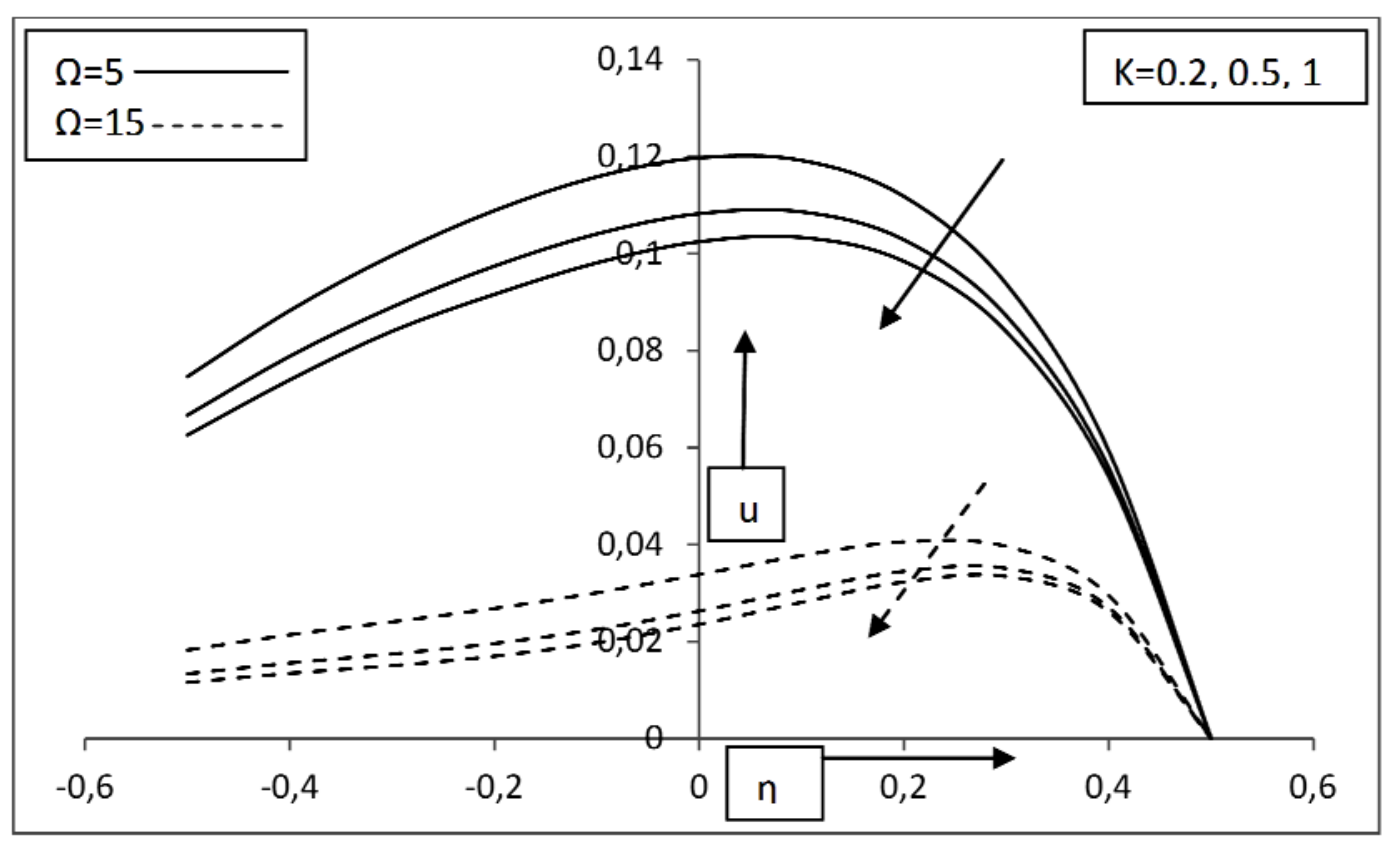

Fig.8.Velocity variations with permeability parameter $K$.

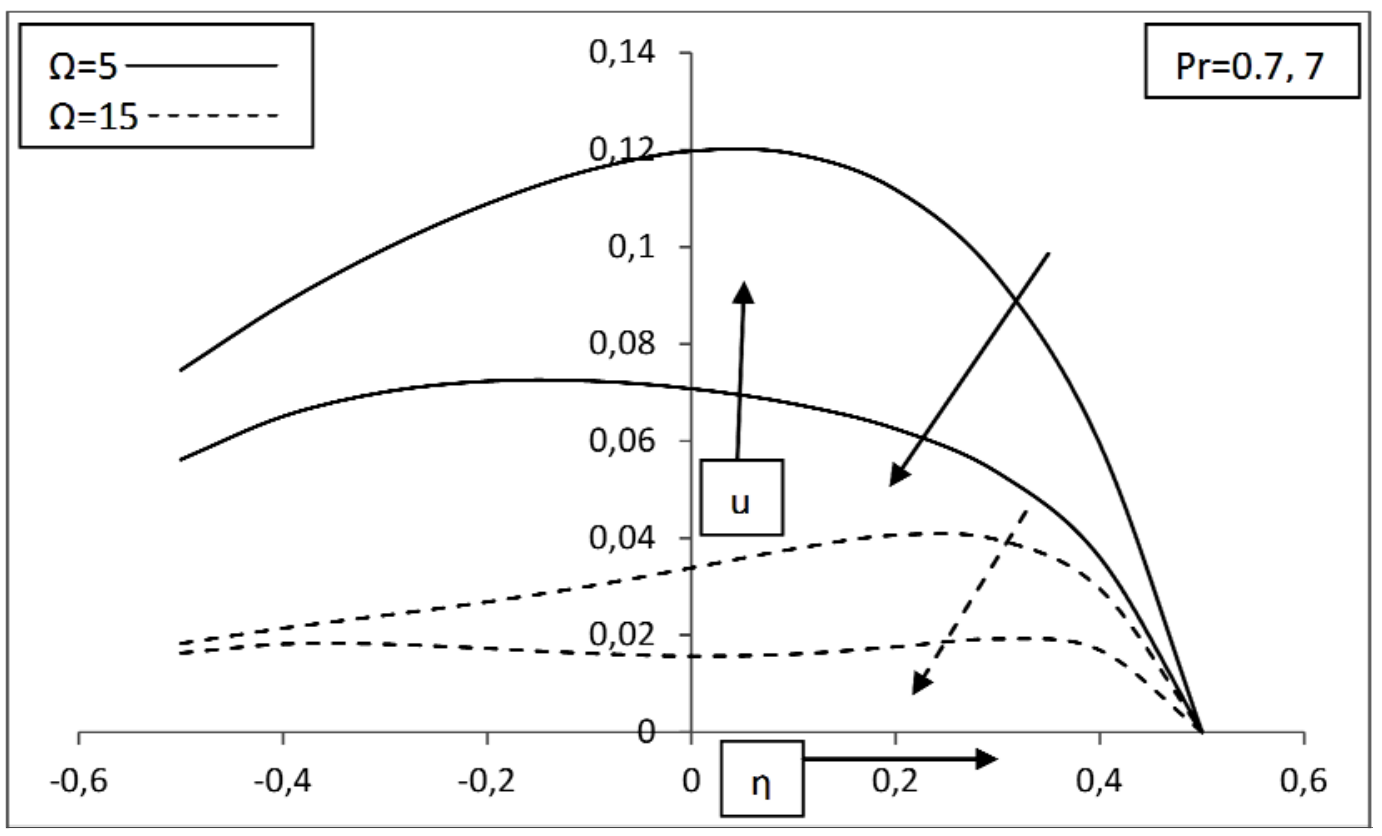

Fig.9.Velocity variations with Prandtl number Pr. 


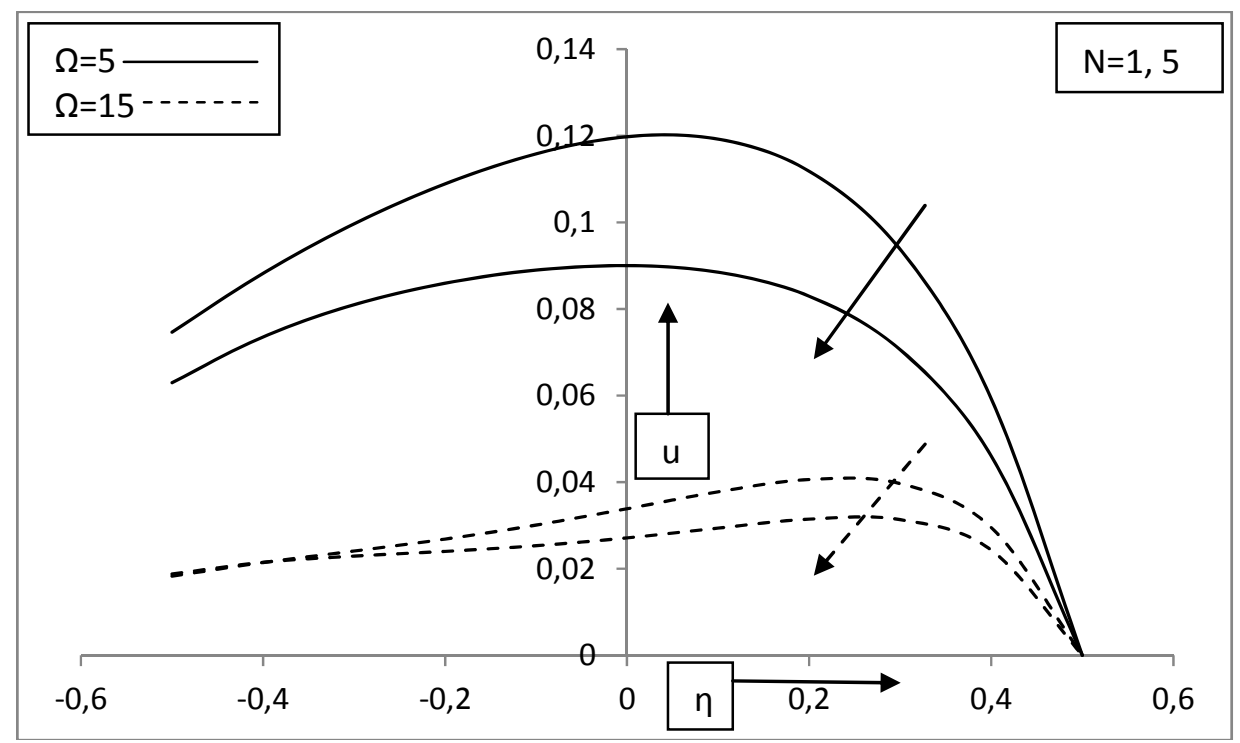

Fig.10. Velocity variations with radiation parameter $N$.

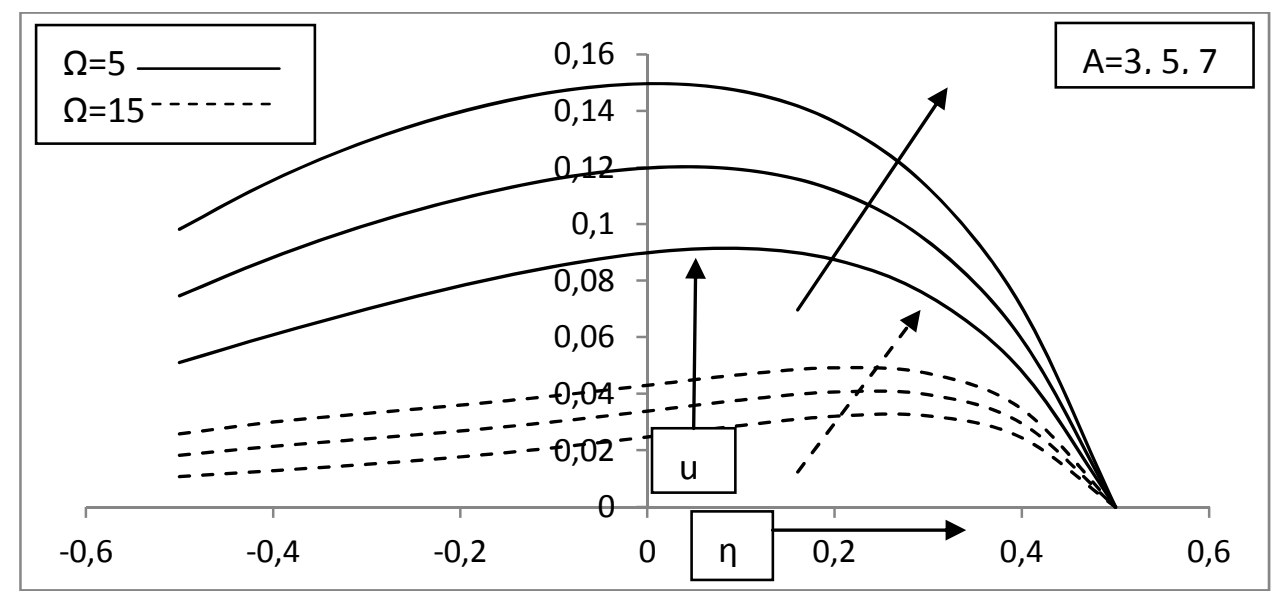

Fig.11. Velocity variations with pressure gradient $A$.

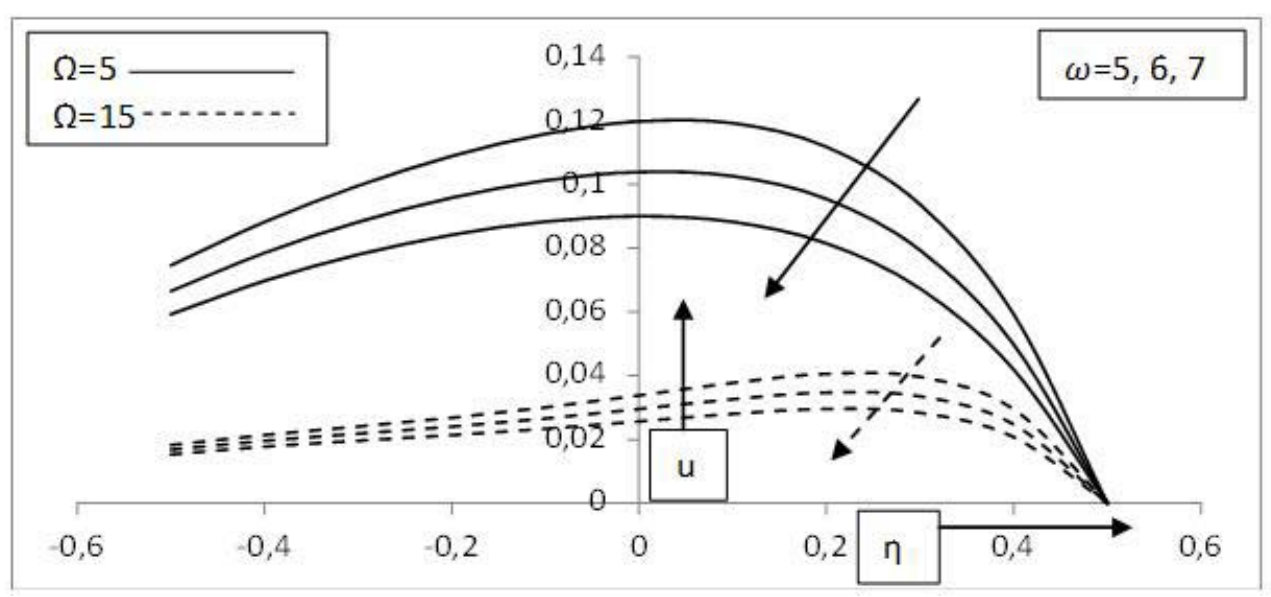

Fig.12. Velocity variations with frequency. 
The skin-friction $\tau_{L}$ in terms of its amplitude $|F|$ and phase angle $\varphi$ has been shown in Figs 13 and 14, respectively. The effect of each of the parameters on $|F|$ and $\varphi$ is assessed by comparing each curve with dotted curves in these figures. In Fig.13 the comparison of the curves V, VI, VIII, and XI with dotted curve I (---) indicates that the amplitude increases with the increase of injection/suction parameter $\lambda$, Grashof Number Gr, permeability of the porous medium $K$ and the pressure gradient parameter $A$. Similarly, a comparison of other curves II, III, IV, VII, IX, X and XI with the dotted curve I (---) reveals that the skin-friction amplitude decreases with the increase of the slip-flow parameter $\delta$, rotation parameter $\Omega$, viscoelastic parameter $\gamma$, Hartmann number M, Prandtl number Pr, radiation parameter $N$. It is obvious that $|F|$ goes on decreasing with the increasing frequency of oscillations $\omega$. From Fig.14 showing the variations of the phase angle $\varphi$ of the skin-friction it is clear that there is always a phase lag, because the values of $\varphi$ remains negative throughout. Here again a comparison of curves III, IV, V, VI and VIII with the dotted curve I (---) indicates that the phase lag increases with the increase of the rotation parameter $\Omega$, viscoelastic parameter $\gamma$, injection/suction parameter $\lambda$, Grashof number $\mathrm{Gr}$ and permeability of the porous medium $K$. A comparison of other curves like VII, IX, X and XI with the dotted curve (---) I shows that the phase lag decreases with the increase of the Hartmann number M, Prandtl number Pr, radiation parameter $N$ and the pressure gradient parameter $A$. For an increasing slip-flow parameter $\delta$ (curves I \& II) the phase lag decreases small values of the frequency of oscillations, $\omega$ but then increases for large values of $\omega$.

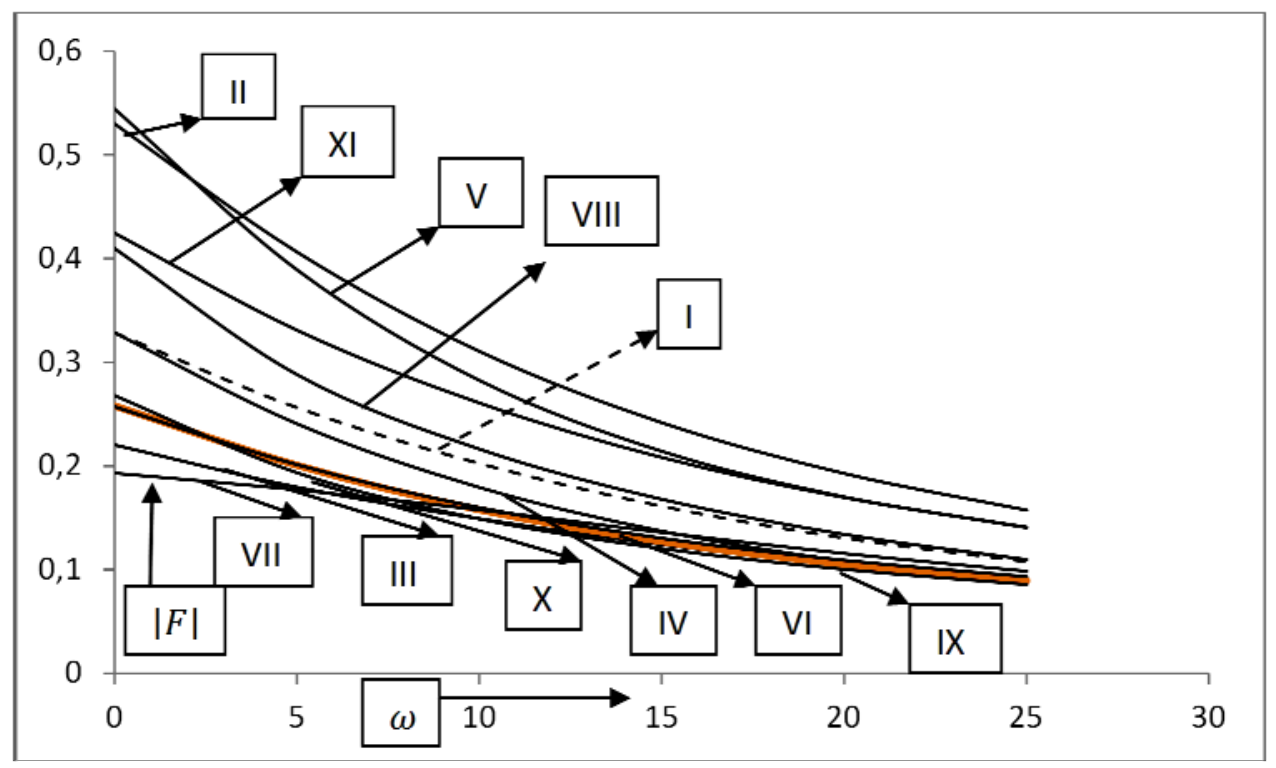

Fig.13. Amplitude of the skin friction. 


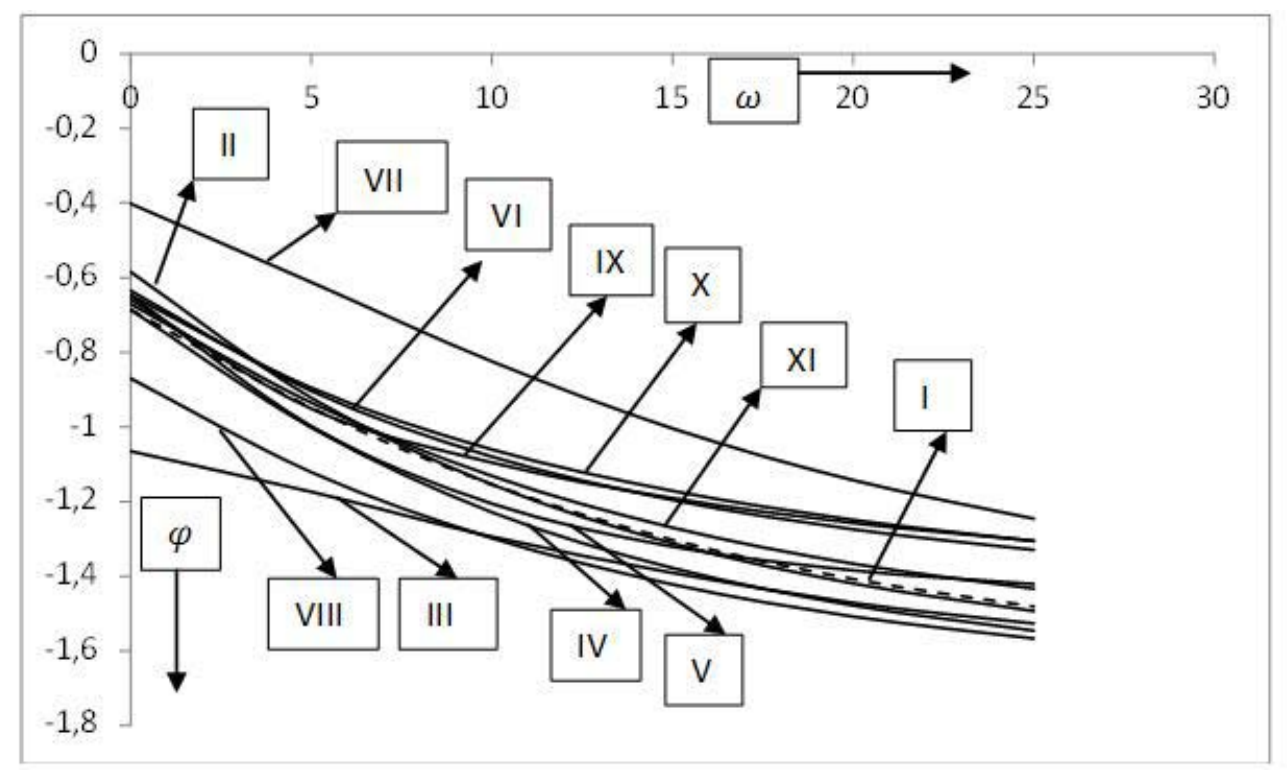

Fig.14. Phase of the skin friction.

\begin{tabular}{|lccccccccll|}
\hline \multicolumn{1}{|c|}{ Values of parameters plotted in Figs $13 \&$ \& 14.} \\
$\delta$ & $\Omega$ & $\gamma$ & $\lambda$ & $\mathrm{Gr}$ & $\mathrm{M}$ & $K$ & $\operatorname{Pr}$ & $N$ & $A$ & Curve \\
0.5 & 5 & 0.2 & 0.5 & 5 & 2 & 0.2 & 0.7 & 1 & 5 & $I(---)$ \\
0.2 & 5 & 0.2 & 0.5 & 5 & 2 & 0.2 & 0.7 & 1 & 5 & $I I$ \\
0.5 & 10 & 0.2 & 0.5 & 5 & 2 & 0.2 & 0.7 & 1 & 5 & $I I I$ \\
0.5 & 5 & 0.3 & 0.5 & 5 & 2 & 0.2 & 0.7 & 1 & 5 & $I V$ \\
0.5 & 5 & 0.2 & 1.0 & 5 & 2 & 0.2 & 0.7 & 1 & 5 & $V$ \\
0.5 & 5 & 0.2 & 0.5 & 1 & 2 & 0.2 & 0.7 & 1 & 5 & $V I$ \\
0.5 & 5 & 0.2 & 0.5 & 5 & 4 & 0.2 & 0.7 & 1 & 5 & $V I I$ \\
0.5 & 5 & 0.2 & 0.5 & 5 & 2 & 1.0 & 0.7 & 1 & 5 & $V I I I$ \\
0.5 & 5 & 0.2 & 0.5 & 5 & 2 & 0.2 & 7.0 & 1 & 5 & $I X$ \\
0.5 & 5 & 0.2 & 0.5 & 5 & 2 & 0.2 & 0.7 & 5 & 5 & $X$ \\
0.5 & 5 & 0.2 & 0.5 & 5 & 2 & 0.2 & 0.7 & 1 & 7 & $X I$ \\
\hline
\end{tabular}

\section{Conclusions}

An oscillatory hydromagnetic convective flow of viscous incompressible and electrically conducting fluid in a vertical porous channel is investigated when the entire system consisting of channel plates and the fluid rotates about an axis perpendicular to the plates. A closed form solution of the problem is obtained. The velocity increases with the increase of the slip-flow parameter $\delta$, injection/suction parameter $\lambda$, Grashof number Gr, Hartmann number M and the pressure gradient parameter $A$. However, the velocity decreases with the increase of the rotation parameter $\Omega$, viscoelastic parameter $\gamma$, permeability of the porous medium $K$, Prandtl number $\operatorname{Pr}$, radiation parameter $N$, and the frequency of oscillation $\omega$. The increase of the Hartmann number i.e., the increasing magnetic field strength, resists the deceleration of the flow due to rotation and the viscoelasticity of the fluid. The amplitude increases with the increase of the injection/suction parameter $\lambda$, the Grashof number Gr, and the pressure gradient parameter $A$. There is always a phase lag of the skin friction. 


\section{Nomenclature}

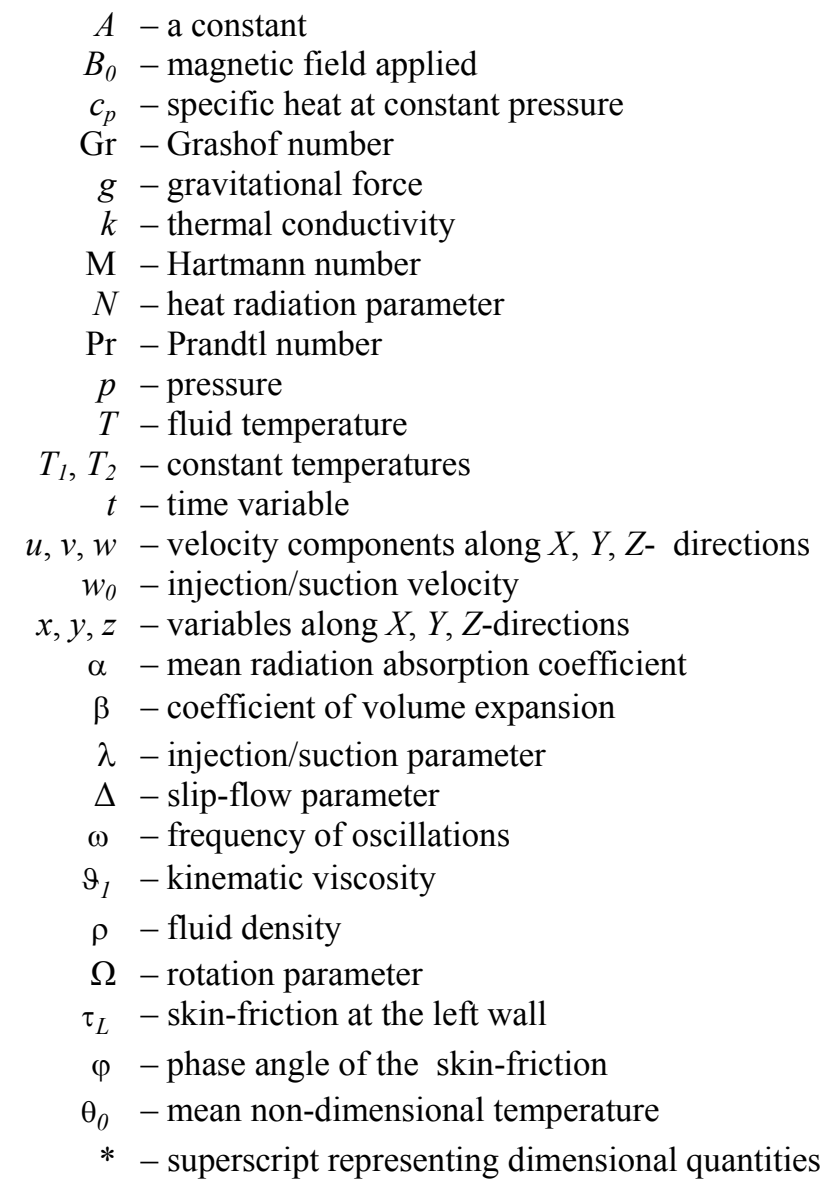

\section{References}

Alagoa K.D., Tay G. and Abbey T.M. (1999): Radiative and free convective effects of a MHD flow through a porous medium between infinite parallel plates with time-dependent suction. - Astrophysics and Space Science, vol.260, pp.455-468.

Attia Hazem Ali and Ewis Karem Mahmoud (2010): Unsteady MHD Couette flow with heat transfer of a viscoelastic fluid under exponentially decaying pressure gradient. - Tamkang J. of Science and Engineering, vol.13, pp.359364.

Bhattacharyya S. and Pal A. (1997): Unsteady MHD squeezing flow between two parallel rotating discs. - Mech. Res. Commun., vol.24, pp.615-623.

Choudhary R. and Das U.J. (2012): Heat transfer to MHD oscillatory viscoelastic flow in a channel filled with porous medium. - Physics Research International, doi:101155/2012/879537.

Cogley A.C.L., Vinvent W.G. and Giles E.S. (1968): Differential approximation for radiative transfer in a non-gray near equilibrium. - American Institute of Aeronautics and Astronautics, vol.6, pp.551-553.

Coleman B.D. and Noll W. (1960): An approximation theorem for functional, with applications in continuum mechanics. - Archive for Rational Mechanics and Analysis, vol.6, pp.355-370. 
Hamaza M.M., Isah B.Y. and Usman H. (2011): Unsteady heat transfer to MHD oscillatory flow through porous medium under slip condition. - Int. J. of Computer Application, vol.33, pp.12-17.

Hamza E.A. (1991): The magnetohydrodynamic effects on a fluid film squeezed between two rotating surfaces. - J. Phys. D: Appl. Phys., vol.24, pp.547-554.

Hamza E.A. (1964): The magnetohydrodynamic squeeze film. - J. Fluid Mech., vol.19, pp.395-400.

Hayat T., Fetecaua C. and Sajid M. (2008): Analytic solution for MHD transient rotating flow of a second grade fluid in a porous space. - Nonlinear Analysis: Real World Applications, vol. 9, pp. 1619-1627.

Hayat T., Javed T. and Abbas Z. (2008): Slip flow and heat transfer of a second grade fluid past a stretching sheet through a porous space. - Int. J. Heat Mass Transfer, vol.51, pp.4528-4534.

Hughes W.F. and Elco R.A. (1962): Magnetohydrodynamic lubrication flow between parallel rotating discs. - J. Fluid Mech., vol.13, pp.21-32.

Kumar A., Varshney C.L. and Sajjan Lal (2010): Perturbation technique to unsteady MHD periodic flow of viscous fluid through a planer channel. - J. of Engineering and Tech. Res., vol.2, pp.73-81.

Maki E.R., Kuzma D., Donnelly R.L. and Kim B. (1966): Magnetohydrodynamic lubrication flow between parallel plates. - J. Fluid Mech., vol.26, pp.537-543.

Makinde O.D. and Mhone P.Y. (2005): Heat transfer to MHD oscillatory flow in a channel filled with porous medium. - Rom. Journ. Phys., vol.50, pp.931-938.

Markovitz H. and Coleman B.D. (1964): Incompressible second order fluids. - Advances in Applied Mechanics, vol.8, pp.69-101.

Marques WJr., Kremer M. and Shapiro F.M. (2000): Couette flow with slip and jump boundary conditions. Continuum Mech. Thermodynam., vol.12, pp.379-386.

Mebine Promise and Gumus Rhoda H. (2010): On steady MHD thermally radiating and reacting thermosolutal viscous flow through a channel with porous medium. - Int. J. of Mathematics and Mathematical sciences, Article ID287435, 12 pages.

Mehmood A. and Ali A. (2007): The effect of slip condition on unsteady MHD oscillatory flow of a viscous fluid in a planer channel. - Rom. Journ. Phys., vol.52, pp.85-91.

Rahmann M.M. and Sarkar M.S.A. (2004): Unsteady MHD flow of viscoelastic Oldroyd fluid under time varying body forces through a rectangular channel. - Bulletin of Calcutta Mathematical Society, vol.96, pp.463-470.

Rajgopal K.R. and Gupta A.S. (1984): An exact solution for the flow of a non-Newtonian fluid past an infinite porous plate. - Meccanica, vol.19, pp.158-160.

Rhodes C.A. and Rouleau W.T. (1966): Hydromagnetic lubrication of partial metal bearings. - J. Basic Eng.-T. ASME, vol.88, pp.53-60.

Singh A.K. and Singh N.P. (1966): MHD flow of a dusty viscoelastic liquid through a porous medium between two inclined parallel plates. - Proc. of National Academy of Sciences India, vol.66A, pp.143-150.

Singh K.D. and Devi R. (2010): Effect of slip velocity on MHD oscillatory flow through porous medium in a channel. International Journal of Physics, vol.3, pp.75-83.

Singh K.D. (2011): Effect of injection/suction convective oscillatory flow through porous medium bounded by two vertical porous plates. - Int. J. of Physical and Mathematical Sciences, vol.2(1), pp.140-147.

Singh K.D. (2012): Viscoelastic mixed convection MHD oscillatory flow through a porous medium filled in a vertical channel. - Int. J. Physical and Mathematical Sciences, vol.3, pp.194-205.

Singh K.D. and Kumar R. (2011): Fluctuating heat and mass transfer on unsteady MHD free convection flow of radiating and reacting fluid past a vertical porous plate in slip-flow regime. - J. Appl. Fluid Mech., vol.4, pp.101106. 
Sivaraj R. and Kumar B. Rushi (2011): Chemically reacting unsteady MHD oscillating slip flow in a planer channel with varying concentration. - Int. J. of Mathematics and Scientific Computation, vol.1, pp.35-42.

Sweet E., Vajravelu K., Robert A. Gorder Van and Pop I. (2011): Analytical solution for the unsteady MHD flow of a viscous fluid between moving parallel plates. - Commun. Nonlinear Sci. Numer. Simulat., vol.16, pp.266-273.

Verma P.D., Sharma P.R. and Ariel P.D. (1984): Applying quasilinearization to the problem of steady laminar flow of a second grade fluid between two rotating porous disks. - J. Tribol. Trans. ASME, vol.106, pp.448-555.

Received: October 25, 2013

Revised: November 28, 2014 